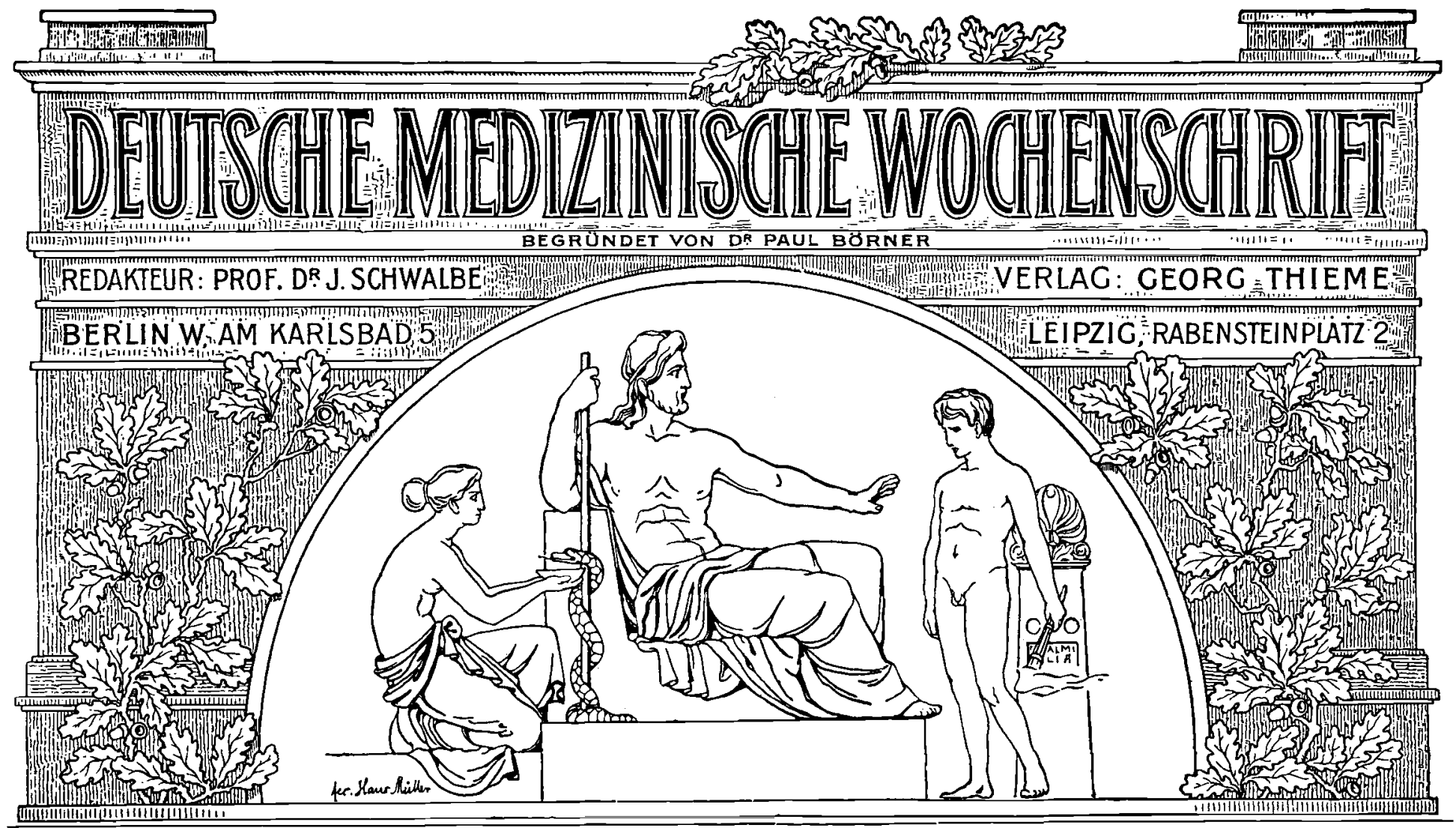

No. 50 .

Berlin, den 10. Dezember 1908.

34. Jahrgang.

\section{Die Behandlung der Neurasthenie.}

\section{Klinischer Vortrag.}

Von Priv.-Doz. Dr. Ełluard Müller in Breslat.

M. H.! Die Neurasthenie ist eine Modekrankheit, keine moderne Krankheit. „Neurasthenie ${ }^{\star}$ ist nur ein neuer Name für ein altes Leiden, das in unserer Zeit, wie man behauptet, eine erschreckende Verbreitung zu gewinnen scheint. Diese Zunahme der Erkrankungen ist aber mehr eine scheinbare als wirkliche. Viel häufiger als das Leiden wird die Diagnose: Neurasthenie. Es handeit sich eben um Dinge, die man früher anders genannt, anders gedeutet und bewertet hat und jetzt mit einem bequemen, bestechenden Schlagwort zusammenfaßt. Dazu kommt eine bessere Kenntnis der nervösen Krankheitserscheinungen; damit steigt für die Statistik rasch die Zahl der Krankheitsfälle. Die Krankheitsziffer schwillt noch mehr durch die gefährliche Neigung unserer Zeit, die Grenzen des Krankhaften mehr als zweckmäßig zu erweitern und vieles mit Neurasthenie zu bezeichnen und selbst zu entschuldigen, was besser als noch normal zu gelten hätte. Anderseits rechnen wir manches wirklich Krankhafte zur Neurasthenie, was andere Ursachen hat und andere Namen verdient.

M. H.! Wenn trotz alledem die Neurasthenie jetzt häufiger wäre als früher, so läge dies kaum an besonderen Schäden des modernen Lebens an sich und kaum an einer fortschreitenden „nervösen Degeneration" unseres Geschlechts. Die großen Massenhysterien des Mittelalters beweisen zur Genüge, daß die abnorme nervöse Konstitution, die gemeinsame Grundlage aller Psychoneurosen, damals mindestens ebenso häufig war wio jetzt. Schon die höhere Geisteskultur bringt es mit sich, daB die nervösen Massenerkrankungen, die es wohl zu allen Zeiten gegeben hat und geben wird, in ihren Erscheinungsweisen sich geändert und die groben Züge mittelalterlicher Hysterie verloren haben. Nervöse Massenerkrankungen werden sich aber mit Vorliebe an Zeiten binden, in denen aufreibende wirtschaftliche und geistige Kämpfe sowie soziale, politische und religiöse Umwälzungen und Wirren unter schneller Aenderung aller äußeren Lebensbedingungen die nervöse Widerstandsfähigkeit eines Volkes zu erschöpfen drohen. In einer solchen Phase stürmischer Entwicklung, die zu schärferer Ausprägung und vorübergehender Häufung abnormer nervöser Zustände neigen mag, leben wir auch jetzt.
M. H.! Die Neurasthenie ist weder eine neue noch eine scharf umschriebene, ganz spezifische Erkrankung. Strenge Grenzen zwischen Nervosität und Neurasthenie gibt es nicht; in zahlreichen Fällen ist selbst die Trennung von der Hysterie nur eine diagnostische Spielerei. Es handelt sich mehr um klinische Typen als um ätiologische Einheiten. Das Bindeglied ist die abnorme, nervöse Konstitution. Sie beruht in der Regel auf angeborener krankhafter Anlage.

Wenn sich aber diese Konstitutionsanomalie vornehmlich in „reizbarer Schwäche des Nervensystems“ äußert, so kommt es zu nervösen Funktionsstörungen, die wir als Neurasthenie bezeichnen. Psychische und körperliche Einzelerscheinungen gruppieren sich dann zu überaus wechselnden klinischen Bildern; ein gemeinsamer charakteristischer Grundzug ist aber die Steigerung der psychischen Erregbarkeit und der psychischen Erschöpfbarkeit. Die Gemütsruhe des Nervengesunden schwindet; es kommt zu psychischem Unbehagen, zu innerer Unruhe, zu krankhafter Gemütsreizbarkeit mit Neigung zu Erregungszuständen, zu Stimmungsschwankungen und vorherrschend depressiven Stimmungslagen und vor allem zu unbegründeten und übertriebenen ängstlichen Befürchtungen. Das Selbstvertrauen geht verloren, die geistige Spannkraft nimmt $a b$, und es entwickelt sich gerne eine krankhafte Willensschwäche. Auf die körperlichen Symptome komme ich noch zurück.

M. H.! Da die seelischen Gleichgewichtsstörungen in der Regel das Krankheitsbild beherrschen, muB die psychische Therapie das beste und natürlichste, ja meist einzig mögliche Heilmittel sein. Diese Ps y ch o th erapie ${ }^{1}$ ) erschöpft sich keineswegs in bestimmten Methoden; Ich erinnere Sie nur an alle günstigen "Imponderabilien", die in der Person des Arztes und seinem Gesamtverhalten gegenüber dem Patienten liegen (Ruf und äußere Erscheinung, sicheres und energisches, aber wohlwollend-freundliches Auftreten etc.). Im allgemeinen kann man aber zwischen einer direkten und einer indirekten seelischen Beeinflussung unterscheiden. Beide Methoden, deren gleichzeitige Anwendung meist geboten ist, bedienen sich im wesentlichen der Suggestion von Besserung und Heilung der Krankheitserscheinungen. Die direkte Suggestion ist die "verbale"; sie gelingt durch das Wort des Arztes und seine Unterhaltung mit dem Patienten. Die indirekte Suggestion bedient sich des Umwegs einer scheinbar körperlichen Behandlung. Sie kann auf die allerverschie-

l) Vgl. Strämpell-Mä 11 er, Psychotherapie in J S chwal bes Handbuch der therapeutischen Technik. Einzelheiten dieses Kapitels sind hier benutzt. 
denste Weise erfolgen. Jede Behandlungsmethode kann bei der Neurasthenie zu Erfolgen führen, weil eben jede Therapie gleichzeitig ein psychisches Heilmittel ist.

M. H.! Den wichtigsten Angriffspunkt für die psychische Therapie bietet die Neigung der Patienten zu ängstlichen Befürchtungen, insbesondere $\mathrm{zu}$ unberechtigter Krankheitsfurcht. Die seelische Beeinflussung beginnt hier mit Aufklärung und Trost auf Grund einer möglichst eingehenden ärztlichen Untersuchung. Größte Genauigkeit dieser Untersuchung ist unerläßlich; nur sie befriedigt den Hypochonder, und nur sie gestattet dem Arzte ein vertrauenerweckendes, sicheres Urteil. Das Verhalten vieler Neurasthenischer ist in der Sprechstunde so charakteristisch, dab es auf den ersten Blick eine richtige Diagnose erlaubt. Dies führt leicht zu Zweifeln an der Realität aller Beschwerden und zu „summarischer“ Untersuchung der Körperorgane. Man vergißt dann leicht, daß auch ein Hypochonder körperlich krank sein kann. Den Patienten quält aber jede tatsächliche oder scheinbare Voreingenommenheit gegenüber seinen Beschwerden; jeder Spott kann ihn tief verletzen. Volles Vertrauen gewinnt er nur dann, wenn der Arzt ihn geduldig anhört und möglichst erschöpfend untersucht. Einer besonders sorgfältigen Prüfung bedarf natürlich das angeblich erkrankte Organ. Eine große Beruhigung liegt oft in der Durchleuch tung der Brustorgane mit Röntgenstrahlen. Anderseits dürfen Sie da, wo nichts ist, nicht allzulange verweilen. Wenn Sie z. B. bei "Phthisiophobie“ die eine Lungenspitze viel länger als die andere beklopfen und behorchen, so schöpft der Kranke oft Verdacht und befürchtet trotz gegenteiliger späterer Versicherung insgeheim eine Erkrankung dieser Seite.

Auf den Nachweis, daß erklärende organische Befunde feblen, folgt in klarer, bündiger Form die feste Versicherung der völligen körperlichen Gesundheit. Der hypochondrische Kranke legt Ihre Worte auf die Goldwage; jede unsichere Ausdrucksweise macht ihn stutzig. Eine nachhaltige Quelle der Beruhigung ist nur seine feste Ueberzeugung von der organischen Gesundheit. Oft ist es nötig, diese Ueberzeugung durch Wiederholung der Untersuchung von neuem zu befestigen.

M. H.! Mit der tröstenden Versicherung, daß das erwartete organische Leiden weder besteht noch droht, dürfen Sie sich nicht begnügen. Sie müssen auch die näheren Ursachen der ängstlichen Befürchtungen festzustellen und zu beseitigen suchen. Wenn z. B. Kranke glauben, an Magenkrebs zu leiden, so können ihre Gründe hierfür sehr verschieden sein. Die einen haben zunächst nur eine unbestimmte Krankheitsfurcht oder ein allgemeines „körperliches“ Mißbehagen, für das sie eine Erklärung suchen. Weil aber Verwandte oder Bekannte an Magenkrebs gestorben sind, befürchten sie das gleiche Uebel. Andere Kranke haben tatsächlich Magenbeschwerden; sie deuten sie nur falsch. Beim Bestehen solcher Organbeschwerden ist aber der intelligentere Kranke mit dem ärztlichen Hinweis, daß ein Magenkrebs nicht vorliegt, kaum zufrieden; er wünscht auch eine seinem Verständnis angemessene Aufklärung über die Natur seiner tatsächlich empfundenen Beschwerden. Die Lösung dieser Aufgabe verlangt einen Arzt, der mit dem Wesen und dem Zustandekommen der körperlichen Symptome bei der Neurasthenie genügend vertraut ist. Wir müssen - nur soviel kann ich hier sagen - zwischen funktionellen und organischen Störungen unterscheiden. Die funktionellen bilden wiederum verschiedene Gruppen; die wichtigsten sind die "psychogenen“. Am klarsten liegen diese Verhältnisse, wenn es sich in letzter Linie um die körperlichen Begleiterscheinungen von Affekten handelt. Jedermann kennt das Erblassen bei Furcht und das Erröten bei Scham und Zorn, das Erbrechen beim Ekel, das Zittern bei Schreck und ähnliche Dinge. Die Neurasthenie erleichtert und steigert solche körperlichen Entladungen seelischer Vorgänge erheblich. Dadurch entwickeln sich gerne scheinbar primäre und scheinbar körperliche Störungen, die dem Kranken als Ursachen seiner seelischen Erregung gelten, während sie doch nur die Folgen sind (Herzbeschwerden, Verdauungsstörungen, vasomotorische Erscheinungen etc.). Zahlreiche sensible Reizerscheinungen wie Parästhesien und Schmerzen entstehen auf ähnliche Weise durch sogenannte Autosuggestion. Schon der Gesunde kann deutlich empfinden, was er sich lebhaft „einbildet“ und ängstlich erwartet. Eine weitere Gruppe funktioneller Störungen erklärt sich jedoch dadurch, daß die abnorme nervöse Konstitution auch andere Teile des Nervensystems betrifft, nicht nur jene Hirngebiete, die den psychischen Leistungen dienen. Beispiele sind die wahren Herzneurosen, die Neuralgien, die echten nervösen Schmerzen sowie das Asthma bronchiale. Auch bei solchen Zuständen erzielt die seelische Beeinflussung oft überraschende Erfolge, sie sind eben der psychischen Bahnung und Hemmung in weitem Maße zugänglich. Selbst organische Symptome können die Folge des nervösen Leidens sein (z. B. starke Abmagerung bei "gastrischer Neurasthenie"); meist beruhen sie jedoch auf komplizierenden andersartigen Leiden. Solche gleichzeitigen organischen Erkrankungen sind bei der Neurasthenie recht häufig und überaus wichtig. Vielfach beobachtet man dann ein schroffes, aber für den Psycho. therapeuten dankbares Mißverhältnis zwischen organischer Gundlage und seelischem Empfinden. Die Heftigkeit aller Schmerzen hängt z. B. nicht nur von der Intensität des auslösenden Reizes, sondern auch von dem Grade der psychischen Empfindlichkeit ab. Gleich schmerzhafte Reize, die der eine leicht erträgt, führen bei dem andern zu lauten Klagen. Ein solches „seelisches Plus" finden wir mit Vorliebe dann, wenn sich das Leiden - z. B. eine Ischias oder eine Tabes dorsalis - bei einem Nervösen entwickelt. Mancher Schmerz besteht dann nur in der Furcht vor dem Schmerz. Der Arzt, der diese Dinge kennt, kann hier durch geschickte Ablenkung und Suggestivmittel oft dasselbe erreichen, was nur durch größere Dosen schmerzstillender Medikamente möglich scheint. Weiterhin pflegt bei solchen Kombinationen die Beurteilung des organischen Leidens eine hypochondrische Färbung anzunehmen (z. B. bei chronischen Geschlechtskrankheiten). Die Beseitigung dieser pessimistischen Auffassung durch Aufklärung, tröstenden Zuspruch und Wiederbelebung der Hoffnung ist oft die beste Arznei. Handelt es sich aber um ein ernstes organisches Leiden, so verlangt die Rücksicht auf die abnorme nervöse Konstitution eine besonders schonende und beschönigende, auf eine genaue Kenntnis der psychischen Eigenart sich stützende individualisierende Aufklärung über den Krankheitszustand. Rücksichtslose Offenheit des Arztes kann hier sehr gefährlich sein. Das Vertuschen hat anderseits seine Grenzen da wo die Rücksicht auf die Behandlungsweise des körperlichen Leidens ein Halt gebietet (z. B. bei den häufigen Kombinationen mit beginnender Tuberkulose).

Mit besonderer Vorliebe soll sich bei Nervösen die Enteroptose finden. Vielfach handet es sich wohl nur um ein mehr zufälliges, belangloses Zusammentreffen, manchmal allerdings auch um engere Wechselbeziehungen. Primär ist gelegentlich das nervöse Leiden; "gastrointestinale“ Neurasthenie kann wohl durch starke Abmagerung zur Wanderniere führen. Nicht selten hat man jedoch tatsächlich den Eindruck, daß beide Affektionen in einer angeborenen, abnormen Anlage eine gemeinsame Grundlage haben und sich dann gegenseitig beein. flussen. Dab aber Wandernieren, Gastroptose, Descensus oder auch Retroflexio uteri Nervengesunde neurasthenisch machen, ist wohl nur ausnahmsweise der Fall. Die Enteroptose gibt in der Regel dem nervösen Zustandsbild nur eine lokale Färbung. Manchmal mag sie auch imstande sein, das nervöse Leiden zu verschlimmern. Die gesteigerte Erregbarkeit der Kranken bezieht sich ja nicht allein auf äußere Reize, sondern auch auf inners Vorgänge unseres Körpers, insbesondere auf sogenannte Organempfindungen. In sehr zahlreichen Fällen ist aber die Enteroptose nichts weiter als eine Verlegenheitsdiagnose. Das Bestreben nach einem körperlichen Ausgangspunkt des Leidens führt zur Suche nach einem kranken Organ. Die Patienten rühmen dann manchmal die Geschicklichkeit des Arztes, der endlich etwas bei ihnen gefunden oder wenigstens diagnostiziert hat. Der Arzt übersieht nur, daß or dadurch die krankhafte Selbstbeobachtung leicht auf eine harmlose, aber nur schwer oder garnicht zu beseitigende körperliche Veränderung lenkt und den Kranken oft erst recht beunruhigt. Am besten ist es, wenn der Patient von dem unerheblichen körperlichen Befund garnichts weib. Halten Sie es 
aber - z. B. mit Rücksicht auf andere Untersucher - für notwendig, ihm dies mitzuteilen, so dürfen Sie keineswegs seinen nervösen Zustand nur damit erklären.

M. H.! Unbegründete Krankheitsfurcht ist nur eine Teilerscheinung der Neurasthenie. Die seelische Beeinflussung erschöpft sich deshalb nicht in Trost und Aufklärung. Sie muß sich mit der ganzen kranken Psyche befassen. Vor allem be. darf die hervorstechende krankhafte Gemütsreizbarkeit der steten Zügelung durch eindringliche Mahnungen zur Selbstbeherrschung. Hausarzt und verständige Umgebung müssen auch hier zusammen wirken; das hemmungslose Sichgehenlassen darf man nicht dulden. Oft empfiehlt sich die Verordnung einer bestimmten geistigen Diät. Die abnorme psychische Erregbarkeit kann die möglichste Fernhaltung von allem, was dem Kranken Sorgen und Aufregungen bereitet, erforderlich machen. Allzulange und allzugroße Behütung schadet aber. Der Nervöse muß allmählich dazu erzogen werden, ein Durchschnittsmal der im Leben unvermeidlichen Bitternisse zu ertragen. Die Welt, in der er arbeiten mub, kennt nicht die Rücksichten, die ihm eine fürsorgliche Familie gewähren kann Das therapeutische Prinzip der Schonung und Uebung gilt auch für die Regelung der geistigen Tätigkeit und der $\mathrm{Be}$ schäftigung des Kranken. Zahlreiche nervöse „Kopfarbeiter" geraten durch verantwortungsreiche und oft nocb sorgenvolle berufliche Anstrengungen in einen von hypochondrischen Zügen freien Zustand hochgradiger „Abspannung“ mit allerlei körperlichen Symptomen wie Kopfschmerz, Schlaflosigkeit und dergleichen. Ein längerer Urlaub erneuert gewöhnlich die geistige Spannkraft. Körperliche Ruhe und möglichste Vermeidung geistiger Tätigkeit sind meist nicht nötig; oft sind sie nicht einmal zweckmäßig. Die beste Erholung dieser Nervösen liegt in der längeren Ausschaltung der erschöpfenden einseitigen Berufstätigkeit. Andere, meist hypochondrische Kranke gefährdet wiederum das Fehlen, nicht das Uebermaß geregelter Tätigkeit. Gegen den Mangel an geistiger und körperlicher Initiative hilft nur die methodische Gewöhnung an Arbeit. Regelmäßige Beschäftigung lenkt gleichzeitig vom eigenen Körper ab; dadurch wird die krankhafte Selbstbeobachtung bekämpft und die Empfänglichkeit für körperliche Beschwerden wesentlich gemildert. Nur eine richtig dosierte, regelmäßige und befriedigende Tätigkeit wirkt aber auf die Dauer günstig. Sie ist oft schwer zu finden bei Kranken, die nur ihrer Gesundheit leben können, und solchen, die einen eigentlichen Beruf nicht besitzen. Wer sich aber ernstlich beschäftigen will, findet im heutigen Getriebe wohl immer ein Feld der Betätigung, das seinen Neigungen entspricht.

M. H.! Der mit den Regeln der psychischen Therapie vertraute Arzt kann die für die allgemeine Praxis ungeeignete Hypnose wohl stets entbehren und bei suggestiblen Kranken auf einfachere und ungefährlichere Weise gleiche Erfolge erzielen. Ein Versuch mit der Hypnose erscheint mir höchstens dann geboten, wenn alle übrigen Hilfsmittel seelischer Beeinflussung erschöpft und resultatlos sind. Auf alle Fälle aber muß sich der Arzt zuvor über ihre Technik und Gefahren genau unterrichten.

M. H.! In zahlreichen Fällen von Neurasthenie ist jede körperliche Behandlung entbehrlich; sie ist in der Regel auch nichts anderes als eine indirekte seelische Beeinflussung. Hierzu stehen Ihnen die verschiedensten Formen körperlicher Therapie zur Verfügung. Für die richtige Auswahl ist im einzelnen Fall vor allem das "nihil nocere“ entscheidend, dann das besondere Vertrauen des Kranken und auch des Arztes zu dieser oder jener Behandlungsweise, ferner die Eigenart des nervösen Zustandsbildes sowie die Berücksichtigung komplizierender organischer Krankheitserscheinungen. Man muf sich jedoch bestreben, den Schwerpunkt der Behandlung möglichst in die direkte seelische Beeinflussung zu verlegen. Die direkten Suggestionen sind zwar schwieriger und zeitraubender, aber dankbarer.

M. H.! Auch die Arzneimittel dienen in erster Linie als Suggestivmittel. Darin liegt eine doppelte Mahnung. Sie müssen stärkere Giftwirkungen möglichst vermeiden und selbst die weitere Darreichung harmloser Mittel dann unterbrechen, wenn ihre Wirkungslosigkeit das Vertrauen des Kranken zur medikamentösen Behandlung erschüttert hat. Zum Verzicht auf größere Dosen differenter Arzneien zwingt schon die lange Krankheitsdauer. Dazu kommt die bedenkliche Neigung der neurasthenischen Kranken, Sklaven ihrer Mittel zu werden Zur Bekämpfung quälender Einzelerscheinungen und vorübergehender Verschlimmerungen des Gesamtzustandes ist allerdings die gelegentliche Verordnung differenter Arzneien - von Morphium und Cocain abgesehen - kaum zu umgehen. Weise Beschränkung ist dann dringend geboten. Die methodische Anwendung des Opiums, die einzelnen Patienten mit schweren Cerebralerscheinungen und Angstzuständen zum Vorteil gereicht, eignet sich z. B. kaum für die ambulante Behandlung Auch das viel gerühmte Codein reicht man am besten nicht länger als einige Wochen (2 bis 4 mal $0,025-0,05$ ). Im allgemeinen empfehlen sich differente Verordnungen nur dann, wenn indifferentere versagen. Dieses Versagen ist aber häufig nur die Folge unzweckmäßiger Auswahl und Darreichung. Als "heilkräftige Arznei“ sollen zunächst nicht ganz wirkungslose "Dinge wie "Mika panis". Aqua destillata und dergleichen verordnet werden. Erfährt ein gebildeter Kranker, sei es auch nur durch das Konversationslexikon oder durch das Schmunzeln eines unvorsichtigen Apothekers, die Eigenart und Wirkungslosigkeit des Mittels, so ist das Vertrauen zum Arzt und damit die Voraussetzung für alle therapeutischen Erfolge geschwunden. Sie wählen deshalb besser unbedenkliche, aber pharmakodynamisch nicht ganz wirkungslose Mittel oder reichen gelegentlich sogar differente Arzneien, aber in möglichst un schuldigen, kleinen Mengen. Sie haben dann gleichzeitig den Vorteil, daß neben der vorherrschenden suggestiven Wirkung eine gewisse physiologische wenigstens möglich ist. Diese gleichzeitige physiologische Wirkung kann bei manchen Mitteln, z. B. bei Bromdarreichungen, die namentlich in Form der brausenden Salze die nervösen Allgemeinsymptome oft vorzüglich beeinflussen, noch wichtiger als die suggestive sein. Man darf ferner dem Kranken nicht solche Medikamente verschreiben, die sich ihm trotz häufigen Gebrauchs schon früher wirkungslos erwiesen haben. Wenn Sie z. B. am Schluß der Konsultation einem Kranken, der zuvor schon bei zahlreichen Aerzten erfolglos "mediziniert" hat, das übliche, ihm wohlbekannte Brom-, Codein- oder Baldrian-Rezept verschreiben, so können Sie den günstigen Effekt ihrer ganzen ärztlichen Beratung ernstlich in Frage stellen. In solchen Fällen müssen Sie eben das alte mindestens in anderer Form oder noch besser etwas anderes verschreiben (Belladonna, Chinin, Nux vomica, Phosphorpräparate, Strychnin, Valylperlen, Validol, bei vasomotorischen Störungen auch Ergotin etc.). Für die Wahl des Medikaments sind oft auch Besonderheiten der körperlichen Störungen entscheidend. Sic werden natürlich bei gleichzeitigen anämischen Zuständen Eisen und Arsenik verordnen (auch in Form der vortrefflichen "tonischen" Pillen (Rp. Acidi arsen. 0,05-0,1, Extr. Strychni 0,5-1,0, Ferri lactici - Chinini hydrochl. ãa 5,0, Extract. Gentian. quant. satis ad pilul. 100; $3 \times$ täglich $1-2$ Pillen). Voraussetzung für die volle Ausnutzung der Suggestivwirkung ist endlich die richtige Darreichung des Mittels. Sie dürfen sich nicht mit dem bloßen Verschreiben begnügen. Sie müssen den Erfolg des „nerven stärkenden“ oder „nervenberuhigenden“ Mittels durch angemessene Verbalsuggestion auch zu begründen oder sogar in sichere Aussicht zu stellen wissen. Auch eine klare, ganz be stimmte Anweisung, wann und wie das Mittel genommen werden muß, ist trotz der etwaigen Indifferenz unerläßlich. Ein Kranker, der merkt, daß es gleichgültig ist, ob er seine Mixtur tee- oder eflöffelweise nimmt, verliert das Vertrauen zu seiner Arznei. So kommt es, dab „dreimal 12“ Tropfen einer Tinktur oft besser wirken als „dreimal 10-20"

M. H.! Die Regelung der Diät verdient genaueste Beachtung überall da, wo eine fehlerhafte Ernährungsweise, ein krankhafter Ernährungszustand oder gar objektive Störungen der Magen-Darmfunktion vorliegen. Eine kräftige, gemischte „Hausmannskost" ist jedoch auch bei nervöser Dyspepsie meist das einzig richtige. Möglichst ausführliche diätetische Vorschriften sind zwar bei solchen Kranken recht beliebt; sie können auch vorübergehend durch ihre Suggestivwirkungen sehr erfolgreich sein. Auf die Dauer konservieren und steigern sie aber die 
hypochondrischen Befürchtungen und damit die Neigung zu einseitiger, falscher Ernährung. In dem Kranken muß die feste Ueberzeugung heranreifen, daß sein Magen-Darnkanal gesund ist und demgemäß alles verträgt, was ein normaler Mensch essen soll und kann. Eingehende diätetische Vorschriften verlangen unter anderem jedoch die häufigen Anomalien der Körperkonstitution. Bei adipösen Kranken sind forcierte Entfettungskuren streng zu vermeiden; sie werden meist schlecht vertragen. $\mathrm{Zu}$ einer langsamen, genügenden Abnahme des Körpergewichts führt im Verein mit größerer Körperbewegung oft schon allein das Verbot allzu üppiger nnd übermäßiger Mahlzeiten. Die stärkere Berücksichtigung vegetabilischer Nahrungsmittel ist namentlich bei gleichzeitiger Stuhlverstopfung zweckmäßig. Bei erheblicher Abmagerung mit schweren neurasthenischen Allgemeinerscheinungen sind anderseits Mastkuren in Verbindung mit Ruhe- und Liegekuren sehr zu empfehlell, auch in Form der Weir-Mitchell-Playfair-Kur (Isolierung des Kranken aus der gewohnten Umgebung, Bettruhe, Darreichung nahrhafter, leicht verdaulicher Mahlzeiten in kleinen, etwa zweistündlichen Pausen, nebenbei milde Massage, Faradisation u. dgl.). Auch die künstlichen Nährpräparate sind dann von Vorteil; schon die Zeitungsreklame sorgt für gleichzeitige günstige Suggestivwirkungen. Alkoholische Getränke darf man jedoch als Suggestiv- und Nährmittel nur ausnahmsweise verordnen. Oft inuß man sogar ihren Genuß auf das erreichbare Minimum einschränken und bei chronischem $\mathrm{Al}$ koholabusus sogar ganz verbieten. Vor vielen, im Volke beliebten „blutbildenden “ und „nervenstärkenden“ ausländischen "Weinen" ist dringend zu warnen. Ihre Echtheit ist ebenso fraglich wie ihr Wert. Man mul überhaupt die gewohnheits. mäßige Zufuhr aller Gifte bei der Neurasthenie verhindern. Die Kranken dürfen deshalb den Alkohol nicht durch reichlichen Teegenuß ersetzen, der ihren Nerven oft viel schädlicher ist als gutes Bier und reiner Wein in mäßigen Mengen. Auch die Harmlosigkeit mancher „alkoholfreien Getränke“, die unsere vielgeschäftige Industrie in geschickter Ausnützung der $A b-$ stinenzbewegung produziert, ist keineswegs sichergestellt. Tabak und Kaffee können ebenfalls schädlich sein. Das Bedürfnis nach Flüssigkeit stillt der Patient am besten durch Wasser sogenannte Mineralbrunnen, natürliche Limonaden, Milch und vielleicht noch durch Kakao.

M. H.! Ein dankbares Feld für ihre Suggestivwirkungen findet die Elektrotherapie in zahlreichen Fällen von Neurasthenie. Die günstige Beeinflussung verstärkt sich noch durch das große Vertrauen zur Elektrizität und durch geschickten ärztlichen Zuspruch während der Sitzungen. Die Tatsache, daß die elektrische Behandlung fast alles durch Suggestion und nur wenig durch den Strom erreicht, rechtfertigt keineswegs eine Geringschätzung der Methodik. Planloses „Daraufloselektrisieren“ kann gegen das "nihil nocere“ verstoßen. Die Exaktheit einer Methode ist ferner ein bedeutsames Hilfsmittel für den suggestiven Erfolg. Sie ist auch Voraussetzung für die Ausnützung jener spezifischen, an sich allerdings unbedeutenden Heilwerte, die man. dem Strom auch bei funktionellen Erkrankungen zuschreibt. Stromesart und Stromesintensität. Dauer und Häufigkeit der Sitzungen verlangen deshalb eine genauere Verordnung. Brüskes Elektrisieren ist zu vermeiden; man muß im Gegenteil - namentlich bei anämischen und erschöpften Kranken - nur schwächere Stromstärken verwenden und überhaupt alles peinlichst vermeiden, was zu Schädigungen führen kann (z. B. rasche Ein- und Ausschaltungen an Stelle des "Einschleichens" und „Ausschleichens“).

Sie können durch allgemeine Faradisation bzw. Galvanisation den ganzen Körper beeinflussen oder durch lokale Einwirkungen die einzelnen örtlichen Störungen und Beschwerden bekämpfen. Als "Tonicum“ gilt mit Recht die allgemeine $\mathrm{Fa}$ radisation (täglich 10-20 Minuten; Pinsel, Bürste oder auch Massierrolle). Sie soll nirgends schmerzhaft sein, an Armen und Beinen jedoch leichte Muskelkontraktionen auslösen. Einige Zeit körperlicher und geistiger Ruhe ist namentlich nach den ersten Sitzungen geboten. Die lokale Behandlung durch vorsichtige Längs- oder Sympathicusgalvanisation richtet sich am Kopfe vor allem gegen "Eingenommenheit", nervöse Schmerzen, Schlaflosigkeit und Schwindel. Auf funktionelle Magen-
Darmbeschwerden sucht man durch Quergalvanisation oder durch Faradisation des Leibes einzuwirken; bei Stuhlträgheit umkreist man mit der elektrischen Massierrolle unter etwas stärkerenı Druck den Leib in der Verlaufsrichtung des Dickdarms etc. Da große und teure, komplizierte und neuartige Apparate oft besser heilen als allbekannte, einfache und billige, so können Sie sich mit Vorteil auch der verschiedenen Formen lıydroelektrischer Bäder, der d'Arsonvalisation sowie der Franklinisation und ähnlichem bedienen.

M. H.! Die viel gepriesene besondere Wirksankeit bestimmter Methoden hydrotherapeutischer und balneologischer Behandlung der Neurasthenie liält sachlicher Kritik kaum Stand. Es fehlen überhaupt noch die wissenschaftlichen Unterlagen für die richtige Abschätzung der physiologischen Beeinflussung des gesunden und kranken Körpers durch diese Therapie. Imnerhin ist uns durch gesicherte Beobachtung am Krankenbett eine Reihe von Erfahrungstatsachen geläufig. So läßt sich z. B. die „erfrischende“, „belebende" und „anregende" Wirkung kurzer kalter Abreibungen und Abklatschungen ebenso wenig leugnen wie die "beruhigende“ durch länger dauernde warme Vollbäder. Die gleichzeitige suggestive Wirkung jeder Methode wird jedoch zugunsten der physiologischen von Hydrotherapeuten und Balneologen stark unterschätzt. Das Vertrauen zur Wasserbehandlung ist ja im Volke weit verbreitet. Die suggestive Wirkung wird noch glößer, je kurmäßiger und präziser sich die Behandlungstechnik gestaltet. Viele Nervöse finden deshalb in gewissen "Kaltwasser-" und "Naturheilanstalten" - wenigstens vorübergehend - eine besonders günstige suggestive Atmosphäre. Schon aus diesen Grüıden rechnet die hydrotherapeutische und balneologische Behandlung zu den besten Hilfsmitteln mittelbarer psychischer Therapie. Weitere Vorteile bietet die oft außerordentlich günstige Beeinflussung des körperlichen Allgemeinbefindens, die gleichzeitige Abhärtung und Hautpflege sowie die Leichtigkeit und Gefahrlosigkeit sachgemäßer Wasseranwendung. Die üblichen Abreibungen und Abklatschungen, die Umschläge und Einpackungen sowie die Bäder und Duschen sind deshalb vorzügliche Heilmittel. Jedes Zuviel ist jedoch von Uebel. Kalte, energische Güsse eignen sich z. B. nicht für blutarme, schwächliche Kranke. Es müssen überhaupt im einzelnen Falle die individuell recht schwankenden Reaktionen auf die Wasseranwelldung ausprobiert und auch späterhin brüske Prozeduren, vor allem mit $\mathrm{zu}$ hohen und $\mathrm{zu}$ niedrigen Temperaturen oder mit großen, häufig wechselnden Temperaturschwankungen vermieden werden. Dab Zusätze zum Wasser, z. B. Kohlensäure und Salzgehalt oder aromatischer Geruch der Bäder, in erster Linie die Suggestivwirkungen verstärken, bedarf wohl kaum der Erwähnung. Selbst von Luft- und Lichtbädern können Sie gelegentlich Gebrauch machen.

M. H.! Auch Heilgymnastik und Massage verdienen gebührende Berücksichtigung. Systematische Leibesübungen z. B. steigern nicht nur die Muskelkraft; sie führen auch zu wachsendem Selbstvertrauen und zur Befriedigung über die geleistete Arbeit. Bei langsamer Steigerung der Anforderungen wird die Körpergymnastik zur Willensgymnastik. Durch eine solche Heilgymnastik werden gleichzeitig Appetit, Stoffumsatz und Schlaf günstig beeinflu@t. Sie ist namentlich zur Bekämpfung neurasthenischer Schwächezustände geeignet. Ueberanstrengung und Uebermüdung sind jedoch streng zu vermeiden. Viele Kranke neigen gerade in dieser Hinsicht zu schädlichen Uebertreibungen, z. B. zu unvernünftigem "Müllern". Die Arbeitsleistungen sind deshalb genauer zu dosieren, zumal manchmal statt körperlicher Bewegung bei sehr geschwächten und blutarmen Frauen möglichste Ruhe geboten ist. Komplizierte Apparate sind meist entbehrlich. Frei-, Hantel- und Stabübungen, einfaches Geräteturnen und dergleichen pflegen vollauf zu genügen. Mit der Kompliziertheit der Apparate, wie wir sie z. B. in "Zandersälen“ finden, steigt natürlich die Suggestivwirkung. Sehr günstig wirkt vielfach auch eine produktive Gymnastik, die eine stete Aufmerksamkeit verlangt (Holzbearbeitung, dosierte Gartenarbeit und dergleichen). Eine vorsichtige Massage ohne allzu energische Muskelbearbeitung ist, namentlich in Verbindung mit Ruhe und Mastkuren, ratsam. Ihre Suggestivwirkung ist ebenso 
wichtig als ihr Einfluß auf Blutzirkulation, Wärmeregulation und Stoff wechsel.

Eine vorzügliche psychische Beeinflussung gelingt häufig durch die sogenannte klimatische Behandlung (Luftkuren, Reisen und dergleichen). Wirksam ist hier vor allem die Ablenkung von dem eigenen Körper durch neue, anregende Eindrücke, die Fernhaltung beruflicher und häuslicher Sorgen und Aufregungen sowie eine bequeme, nur der Gesundung dienende Lebensweise. Sie schlagen am besten dem Kranken einen bestimmten Kurort vor. Für seine Auswahl sind aber die klimatischen Verhältnisse nur dann entscheidend, wenn Körperkonstitution oder komplizierende Erkrankungen wie Arteriosklerose, Asthma und Herzleiden eine besondere Berücksichtigung verdienen. Eine spezifische Beeinflussung der Neurasthenie durch das Klima ist nicht zu erwarten. Der körperlich normale Neurasthenische kann ebenso in Seeluft wie in Gebirgsluft gesunden. Die Hauptsache bleibt, daß der Kurort den individuellen Neigungen des Kranken für Gebirge oder See entspricht und ihm gleichzeitig eine zusagende und seinen ökonomischen Verhältnissen entsprechende äußere Lebensführung bietet. Die besten klimatischen Verhältnisse eines Kurortes können nutzlos sein, wenn den emplindlichen Nervösen dort die Mücken plagen! Vorzüglich wirken auf Körper und Geist oft Winterkuren im Gebirge, wo der Kranke viel Gelegenheit zu gesundem Sport und fleißiger Bewegung in frischer Luft findet. Anstrengende Reisen mit häufigem Wechsel des Aufenthaltsortes können anderseits schädlich sein; sie erschöpfen den Kranken manchmal erst recht.

M. H.! Gegen Neurasthenie hilft also nicht ein einzelnes Heilmittel, sondern nur eine den individuellen Verhältnissen angepaßte Kur, die unser gesamtes Rüstzeug psychischer und körperlicher Beeinflussung berücksichtigen muß. Eine solche methodische und genügend lange fortgesetzte Behandlungsweise gelingt am leichtesten in gut geleiteten Kuranstalten; sie eignen sich besonders für schwerere Fälle sowie für unschlüssige und energielose Kranke. Heilanstalten schützen den Patienten durch die Isolierung aus dem früheren Wirkungskreis vor ungünstigen äußeren Einwirkungen und gewähren dem Arzte die Möglichkeit $\mathrm{zu}$ anhaltender persönlicher Beeinflussung und genauer Kontrolle über die ganze Behandlungsweise. In den Sanatorien herrscht außerdem eine vielfach günstige therapeutische Vielgeschäftigkeit, die sich allerdings nicht in steten Aenderungen der Behandlungsmethode äubern soll.

M. H.! Eine erfolgreiche Behandlung der Neurasthenie ist unmöglich, wenn der gute Wille, gesund zu werden, fehlt. Heilungen sind deshalb so selten in Fallen "traumatischer Neurasthenie", wo die Aussicht auf Rente nicht nur die Krankheit entstehen läßt, sondern auch die Heilung verhindert. Hier ist in der Regel keine intensive, sondern eine möglichst knappe und häufig sogar gar keine Behandlung angezeigt. Zwecklos sind vor allem fast alle Heilverfahren, die gegen den Willen oder ohne besondere Neigung des Kranken erfolgen. Die Aufgabe, solche Nervöse „durch Arbeit zur Arbeit“ zu erziehen, werden auch besondere Heilstätten für Unfallkranke nur selten lösen; derartige Heilstätten werden überhaupt schon durch die ausgiebige Möglichkeit zu psychischer Infektion - leicht zu Brutstätten nervöser Beschwerden. Nur eine geeignete Prophylaxe der Aerzte sowie Aenderungen der Versicherungsgesetze können helfen. Zunächst einmal ist die Abkürzung und Vereinfachung unseres komplizierten Schadenersatzverfahrens mit möglichst gerechter, aber rascher und entgültiger Erledigung des Rentenkampfes anzustreben. Auch eine weit verbreitete Pseudohumanität, die allzu leicht allzu hohe Renten gewährt, ist nicht schuldlos. Sie verhindert die heilkräftige allmähliche Wiederaufnahme geregelter Beschäftigung und steigert die Unzahl der Kranken mit „traumatischer Neurose" durch die verführerisch leichte Aussicht auf Rente. Oft ist „krankhafte Willensschwäche" nur ein beschönigendes Wort für das „nicht arbeiten wollen“. Wir sehen in unserer Poliklinik fast täglich Kranke aus der körperlich arbeitenden Bevölkerung, die trotz erheblicher nervöser Beschwerden zum Vorteil ihres Gesundheitszustandes - volle Arbeit leisten, weil sie eben arbeiten wollen und müssen. Auch sonst muß sich die ärztliche Beurteilung der traumatischen Neurasthenie ändern. Wenn relativ geringfügige psychische Traumen imstande waren, schwere nervöse Erscheinungen mit lange dauernder erheblicher Beeinträchtigung der Erwerbsfähigkeit auszulösen, so fehlte eben dem Kranken von vornherein jenes Maß nervöser Widerstandsfähigkeit, das wir in unseren Betrieben, genau so wie eine gewisse körperliche Widerstandsfähigkeit, verlangen müssen. Wenn aber nur der Kampf um die Rente zu abnormer nervöser Verfassung geführt hat, so scheint mir die Entschädigungspflicht mindestens fraglich. Die Prophylaxe verlangt auch, daß der Arzt alle schädlichen Suggestionen vermeidet. Intensive Behandlung nervöser Unfallsbeschwerden suggeriert z. B. eine besondere Wichtigkeit derselben. Die möglichst sorgfältigen Bemühungen, im einzelnen Falle die Neurose auch objektiv zu beweisen (z. B. durch die beliebten, aber meist nichtssagenden konzentrischen Gesichtsfeldeinschränkungen, Abschwächungen des Hautsgefühls etc.) sind weiterhin die wichtigste Quelle für viele suggestiven Kunstprodukte, die als „objektive" Zeichen seines Leidens dem Kranken im Rentenkampf sehr willkommen sind.

M. H.! Wie sich nun im einzelnen Fall ein aussichtsvoller Kurplan gestaltet, soll bei einigen praktisch wichtigen Zustandsbildern der Neurasthenie noch kurz besprochen werden.

M. H.! Die zielbewuBte Behandlung der sexuellen Neurasthenie hat zur Voraussetzung eine genaue Kenntnis der Krankheitsursachen im einzelnen Fall. Meist wird das Leiden auf sexuelle Ausschweifungen zurückgeführt; häufig sind auch chronische Erkrankungen der Sexualorgane ätiologisch bedeutsam. Unter den geschlechtlichen Ausschweifungen steht voran die Onanie. Bei neuropathischer Veranlagung wird sie oft frühzeitig, ja schon vor der Geschlechtsreife begonnen, allzuhäufig getrieben und lange Jahre fortgesetzt. Leicht entwickeln sich dann neben allgemeinen neurasthenischen Symptomen quälende örtliche Beschwerden im Bereich des Urogenitalapparates (Parästhesien, häufige und schwächende Pollutionen; Auftreten derselben schon bei ganz geringer mechanischer Reizung und selbst ohne besondere sinnliche Erregung; ungenügende, zu kurz oder zu lange dauernde oder gar keine Erektionen und dergleichen).

Schon bei der ersten Untersuchung muß sich der Psychotherapeut das Vertrauen des Kranken sichern. Er mub aus verschleiernden Schilderungen und vagen Andeutungen das Uebel rasch erkennen und dem Kranken die befriedigende Aussprache möglichst erleichtern. Auf die eingehende ärztliche Untersuchung folgt auch hier die bestimmte Versicherung, daß das befürchtete organische Nervenleiden fehlt und überhaupt niemals durch Onanie allein entsteht. So gelingt es, die übertriebenen und durch die verderbliche Lektüre gewisser populär-medizinischen Bücher genährten Befürchtungen zu beseitigen. Auch die moralischen Gewissensbisse sind auf das richtige Maß zu beschränken. Oft ist hier „sexuelle Aufklär'ung" am Platze. Manche sehen z. B. in der Pollution an sich ein bedenkliches Krankheitssymptom, während sie doch beim geschlechtsreifen, abstinenten Menschen nur ein physiologischer Notbehelf ist. Andere Onanisten halten nur sich für die größten Sünder, weil sie nicht wissen - dies gilt übrigens auch für viele Aerzte - , daß wohl fast alle gesunden jungen Leute gelegentlich einmal onanieren (wenn auch nicht immer in Form der üblichen Masiurbation).

Auch bei jener Form sexueller Neurasthenie, die mit chronischen, insbesondere gonorrhoischen Erkrankungen der Sexualorgane zusammenhängt, ist die psychische Allgemeinbehandlung meist ebenso wichtig wie die lokale Therapie. Schon während der ersten Lokalbehandlung ist der Arzt verpflichtet, durch beruhigenden Zuspruch und Aufklärung den schweren nervösen Erscheinungen vorzubeugen, die sich durch einseitig spezialistische Behandlung von Geschlechtskrankheiten bei Neuropathen mit Vorliebe entwickeln.

M. H.! Dauernde Erfolge sind bei sexueller Neurasthenie meist nur durch die weitere Regelung des sexuellen Lebens $\mathrm{zu}$ erzielen. Anfänglich muß der Kranke von allen sexuellen Dingen möglichst abgelenkt werden (regelmäßige Beschäftigung, geeigneter Sport u. dgl.). Eingehende „Lokalbehandlung“ ist deshalb eher schädlich als nützlich. Man unterstützt besser die erwähnte seelische Beeinflussung durch Behandlung 
des Allgemeinzustandes mit Diät, Elektro- und Hydrotherapie, Regelung des Stuhlgangs, Brom u. dgl. Nur für leichtere Fälle psychogener Impotenz eignen sich von vornherein die suggestiven Hilfen durch Aphrodisiaca (Yohimbin etc.). Die ärztliche Entscheidung darüber aber, wie der erwachsene Nervöse nach Besserung der örtlichen Beschwerden seine physiologisch durchaus berechtigte Libido befriedigen soll, ist ungemein schwierig. Auf Körper und Geist des gesunden Erwachsenen wirkt eine mäßige und natürliche sexuelle $\mathrm{Be}$ friedigung unzweifelhaft günstig. Völlige Abstinenz $\mathrm{kann}$ anderseits unschädlich sein. Im allgemeinen ist sie es hingegen nicht, wenn auch die Schäden nur selten schwer und offenkundig sind. Daß viele körperlich gesunde Neurasthenische unter erzwungener Abstinenz aber direkt leiden, beweist die klinische Erfahrung. Die ärztliche Empfehlung der Ehe ist nun ein heikel Ding. Ein gutes Heilmittel für Nervöse ist nur eine glückliche Ehe; dazu gehören Voraussetzungen, die sich dem Machtbereich des Arztes entziehen. Bei fraglicher Potenz kann sich zudem durch die Furcht vor dem Fiasko die Nervosität so verschlimmern, daß die ärztliche Heiratslizenz fraglich wird. Noch ernster wird die Situation, wenn sich zwei Neurasthenische in der Ehe treffen und der weibliche Teil es an verständnisvoller Nachsicht fehlen läßt. Die Nachteile des außerehelichen Verkehrs sind anderseits gerade für Neurasthenische häufig größer als die Vorteile (Furcht vor Ansteckung, gleichzeitiger Alkoholmißbrauch, die notwendige Heimlichkeit mit Ungunst von Ort und Zeit, Beeinträchtigung des sinnlichen Genusses durch die Vorbeugung von Infektion und Schwangerschaft, sittliche Bedenken u. dgl.)

Sie sehen, meine Herren, daß die sachgemäße Behandlung der sexuellen Neurasthenie mehr ist als ein einfaches ärztliches Problem. Wir müssen hier mit vielgestaltigen sozialen Verhältnissen, mit widersprechenden Lebensanschauungen sowie mit den wechselnden Lehren der Ethik und der Konfessionen rechnen. Als Aerzte müssen wir aber daran mitarbeiten, dal wir zwischen sexueller Prüderie und Sittenlosigkeit allmählich einen gesunden Mittelweg gewinnen, der durchaus berechtigten, natürlichen Instinkten gebührend Rechnung trägt.

M. H.! Wenn die Herzneurasthenie, wie so häufig nur, auf der Furcht vor einem Herzfehler beruht, so genügt oft schon die feste Versicherung, dal ein organischer Fehler trotz genauester Untersuchung nicht nachweisbar ist, im Verein mit angemessener Aufklärung über den psychogenen Ursprung der Störung zur Besserung und Heilung (Hinweis auf das physiologische Herzklopfen bei seelischer Erregung, dann auf die fehlerhafte Deutung dieser Erscheinung durch den Kranken infolge Verwechselung von Ursache und Wirkung und endlich auf seine gesteigerte Empfindlichkeit infolge der krankhaften Selbstbeobachtung). Als unterstützende Mittel gelten: Umschläge auf die Herzgegend, spirituöse Abwaschungen derselben, kohlensaure Bäder, Brom- und Baldrianpräparate sowie die Vermeidung etwaiger Stuhlträgheit und Magenüberfüllung. Schwieriger gestaltet sich die Sachlage bei den echten Herzneurosen oder gar bei komplizierenden organischen Erkrankungen. Trotzdem spielt auch hier die psychische Therapie eine bedeutsame Rolle. Bei neurasthenischen Kranken mit Klappenfehlern gelingt es z. B., die übertriebenen Befürchtungen nicht selten durch den Satz zu bekämpfen, daß die Zukunftsaussichten weniger von dem geschädigten Klappenapparat an sich als von dem Zustand des noch ganz leistungsfähigen Herzmuskels abhängen. Komplizierende Herzleiden verlangen ferner die möglichste Fernhaltung stärkerer psychischer Erregungen und die Bekämpfung der auffälligen Reizbarkeit, die gerade solche Kranke häufig zeigen.

Die neurasthenischen $\mathrm{S}$ chlafstörungen beruhen häufig nur darauf, daß die Furcht vol der Schlaflosigkeit das Einschlafen erschwert. Nicht selten ist auch der. Schlaf zu kurz, zu leise und unruhig oder allzu bleiern-schwer. Jedenfalls fehlt beim Erwachen das beglückende Gefühl wiedergewonnener körperlicher und geistiger Frische. Das schematische Verschreiben von Schlafmitteln pflegt den Zustand nur zu verschlimmern; meist ist die Entziehung der Schlafmittel, nicht ihre Verordnung geboten.

Ist bei nervöser Schlaflosigkeit nur das Einschlafen er- schwert. so verbinden Sie mit dem Hinweis auf den psychogenen Ursprung der Störung und auf die Gefahr, sich den Schlaf durch stärker wirkende, auf die Dauer schädliche Präparate auf Kosten der Gesundheit zu erkaufen, eine indirekte seelische Beeinflussung durch hydriatrische Maßnahmen oder durch relativ unschuldige Arzneimittel (neben kurzen kalten Fußbäder’n, Umschlägen und Einpackunngen kleine Bromdosen, eine Tasse Baldriantee, bei zuvor nicht Abstinenten selbst ein Gläschen Bier und dergleichen). Gleichzeitig erfolgt die Mahnung zu strenger Regelung der Lebensweise (vor allem Aufstehen und Schlafengehen zu ganz bestimmten, frühen Stunden; Vermeidung größerer Mahlzeiten, erregender Getränke und ungeeigneter Lektüre am Abend sowie an Stelle später geistiger Anstrengung eine mäßig ermüdende körperliche $\mathrm{Be}$ wegung). Jedenfalls müssen Sie versuchen, ohne Hypnotica auszukommen. Scheinen sie unentbehrlich, so darf man sie nur vorübergehend und in längeren Pausen reichen. Die Neurasthenie ist eben ein chronisches Leiden und die Schlafstörung oft ein hartnäckiges Symptom. Unter den Schlafmitteln empfiehlt sich noch am meisten das Paraldehyd. Die Gefahr del Gewöhnung scheint mir hier am geringsten. Abwechselung ist stets geboten. Man verschreibt dann: Chloralamid, Amylenhydrat, Veronal, Hedonal, Trional, Proponal, Isopral, Neuronal, Dormiol etc. Präparate, die sich schon früher einmal als wirkungslos erwiesen haben, scheiden am besten aus. Patienten, die auch mit Schlafmitteln niemals mehr zu schlafen glauben, können Sie vorübergehend auch ein zuverlässiges Hypnoticum verordnen, um sie durch den Beweis des Gegenteils psychisch zu beruhigen. Ersetzen Sie späterhin das Medikament geschickt durch ein Suggestivmittel, oder erklären Sie von -vornherein, daß sich nach mehrmaliger Darreichung dauernder, spontaner Schlaf einstellen wird, so erzielen Sie nicht selten erfreuliche Erfolge.

Als kurzes Beispiel für die psychische Behandlung der sogenannten Phobien mag die Platzangst dienen. Der Kranke muß durch sorgfältige ärztliche Untersuchung, tröstenden $\mathrm{Zu}$ spruch und Aufklärung die feste Ueberzeugung der organischen Gesundheit gewinnen und wissen, daß Zittern, Schwäche, Ohnmacht, Herzklopfen und alle scheinbar „körperlichen“ Störungen an sich unbedenklich und nicht die Ursache, sondern die Folgen seiner ängstlichen Erregung bei dem Ueberschreiten des Platzes sind. Auch ein arzneiliches Suggestivmittel, das kurz zuvor genommen wird, gibt gerne das nötige Selbstvertrauen wieder. Die Störung wird oft zweckmäßig durch eine langsame Steigerung der Anforderung überwunden, indem man z. B. den freien Platz anfänglich nur peripher umschreiten, dann den Kranken beim Gang über den Platz durch einen eingeweihten, energischen Erwachsenen, später durch ein Kind begleiten läßt etc.

M. H.! Noch einige Bemerkungen zur Prophylaxe. Möbius sagte einmal: Will man den besseren Menschen, so muß man ihn zeugen, nicht nur erziehen. Auch bei Prophylaxe der Neurasthenie ist die Beseitigung aller Schäden, die zu den Ursachen der angeborenen neuropathischen Anlage rechnen, nicht minder wichtig als die vorbeugende Behandlung der gefährdeten Kinder. Die allgemeine Bekämpfung dieser abnormen Anlage erfordert jedoch Maßnahmen, die die Grenzen ärztlichen Handelns weit überschreiten. Praktisch erreichbar ist meist nur eine individuelle Prophylaxe durch die gegenseitige Unterstiitzung von Arzt, Elternhaus und Schule.

Wenn nervöse Eltern nervöse Kinder haben, so liegt dies oft mehr an dem schlechten Beispiel und der falschen Erziehung als an der fehlerhaften Anlage. Sie müssen die Nervosität der Eltern mitbehandeln, wenn das nervöse Kind gesunden soll. Nur dann gelingt es, das Seelenleben des Kindes in richtige Bahnen zu lenken. Die krankhafte Reizbarkeit erfordert schon frühzeitig eine wohlwollend-freundliche, aber energische Anleitung zu psychischer Hemmung. Schreckhaftigkeit und Aengstlichkeit verlangen die Erziehung zum Mut, vor allem durch Turnen, Spiel und vernünftigen Sport. Die geistige Erschöpfbarkeit warnt vor zu früher und zu ausgiebiger Anspannung der Geisteskräfte. Damit ist die überaus wichtige Frage der "geistigen Ueberbürdung" angeschnitten. Der Schule wird hier vieles zur Last gelegt, was Eltern und Kinder selbst verschulden (u. a. Verkennung der Begabung mit 
falscher Wahl der Lehranstalt, stete Anspornung zu kaum erreichbaren und nicht obligatorischen, auch künstlerischen Leistungen, andere ungünstige Momente wie nervöses häusliches Leben, fehlerhafte Ernährung, unge日ignete Vergnügungen und ausartender Sport, schlechte Lektüre, Masturbation und selbst Alkohol und andere Gifte). In mancher Hinsicht sündigt freilich auch die Schule, aber weniger durch die Größe des Pensums als durch die Art des Unterrichts und durch die Person des Lehrers. Nervosität des Lehrers macht auch das Kind nervös. Schädlicher als die Vorbereitung ist die Furcht vor dem Unterricht.

Die Fürsorge für das nervöse Kind darf nicht in Verweichlichung ausarten. Unsere Jugend braucht auch geistige Abhärtung, nicht nur körperliche; Muttersöhnchen, bei denen die Verweichlichung schon in der Wiege beginnt, stranden meist an den Klippen des modernen Lebens. Trotz straffer, aber von Härte freier häuslicher Disziplin müssen die Kinder so früh wie möglich zu eigener Verantwortlichkeit und selbständigem Handeln erzogen werden. Das nervöse Kind bedarf zwar des ärztlichen Schutzes, wenn Eltern und Lehrer ihm das als Sünde anrechnen, was wirklich krankhaft ist. Dem Kinde gegenüber muß man aber die Grenzen des Pathologischen möglichst enge ziehen. Die Nervosität darf ihm nicht als stets willkommene Entschuldigung für Dinge dienen, die an sich vielleicht krankhaft, aber der psychischen Beeinflussung durch die Erziehung zugänglich sind. Nervöse Kinder bedürfen ebenso wie der kranke Erwachsene der Schonung, aber auch der methodischen Gewöhnung an regelmäßige Arbeit und selbst an die rauhen Seiten des Lebens. Jede verhätschelnde Aengstlichkeit bei den kleinen Unfällen und Uebeln der Kinder ist gefährlich; sie erzieht geradezu zu krankhafter Selbstbeobachtung, zu Empfindlichkeit und Uebertreibung. Späterhin ist richtige Berufswahl von größter Bedeutung; man muß hier weniger Rücksicht auf Eitelkeit und materielle Gesichtspunkte als auf Begabung und Neigung nehmen.

Mit der Geistespflege des nervösen Kindes, die die ganze moderne Erziehungslehre umfaßt, muß eine sachgemäße Körperpflege Hand in Hand gehen. Die Magerkeit und Blässe der nervösen Kinder, ihre Magen- und Darmbeschwerden sowie ihre Neigung zu Kopfschmerzen, Ohnmachten, Schlafstörungen und ähnlichem bieten außerdem Angriffspunkte für eine gleichzeitige diätetische, physikalische und nedikamentöse Therapie.

M. H.! Die Fülle wichtiger prophylaktischer und therapeutischer Gesichtspunkte ist bei der Neurasthenie so groß, daß ich hier nur die Richtlinien für unser ärztliches Handeln wiedergeben konnte. Wenn es aber trotz aller Behandlungsweisen meist mißlingt, die Kranken ganz und dauernd zu heilen, so liegt dies eben daran, daß die Neurasthenie ein chronisches Leiden ist und in der Regel auf einer therapeutisch unzugänglichen, abnormen nervösen Veranlagung beruht. Die Ursachen dieser abnormen Anlagen sind jedoch nicht allein Krankheiten im engeren Sinne, sondern auch soziale Schäden verschiedenster Art. Die nervöse Gesundung unseres Geschlechts hat deshalb zur Voraussetzung die soziale Gesundung. Zum Kampfe gegen alle Nervosität ruft aber schon die Tatsache, daß ihre Gefahren für die menschliche Gesellschaft wohl kaum geringer sind als diejenigen der anerkannten Volksseuchen; sie sind nur durch Ziffern schwerer wiederzugeben und zu beweisen. 